\title{
020704
}

\section{The Visual Localization of the Centre of Triangles in Young Children and Adults}

\section{Gabriel Baud-Bovy and Edouard Gentaz}

\section{OpenEdition}

\section{Journals}

Electronic version

URL: http://journals.openedition.org/cpl/430

DOI: $10.4000 / \mathrm{cpl} .430$

ISSN: $1379-6100$

Publisher

Centre PsyCLÉ

\section{Electronic reference}

Gabriel Baud-Bovy and Edouard Gentaz, «The Visual Localization of the Centre of Triangles in Young Children and Adults », Current psychology letters [Online], 13, Vol. 2, 2004 | 2004, Online since 02 July 2004, connection on 08 September 2020. URL : http://journals.openedition.org/cpl/430 ; DOI : https:// doi.org/10.4000/cpl.430

This text was automatically generated on 8 September 2020

(c) All rights reserved 


\title{
020704
}

\section{The Visual Localization of the Centre of Triangles in Young Children and Adults}

\author{
Gabriel Baud-Bovy and Edouard Gentaz
}

Received November 25, 2003

2 Revision June 1, 2004

3 Accepted June 3, 2004

4 On line July 2, 2004

Introduction

5 For most 2D shapes, the centre is an ill-defined geometrical notion. For example, the centre of mass, the orthocentre, and the centres of inscribed and circumscribed circles are different for most triangles. This ambiguity raises the question of the location of the perceptual centre when localizing the centre of planar shapes. In adults, several studies have shown that the perceptual centre is close to the centre of mass of the shape (e.g. Beghi, Vicario, \& Zanforlin, 1983; Davi, 1989; Proffitt, Thomas, \& O’Brien, 1983; Vos et al., 1993). Similar results have been observed when participants were asked to estimate the position of the "equilibrium point" (centre of mass) of a shape (BaudBovy \& Soechting, 2001; Bingham \& Muchisky, 1993ab). The importance of the centre of mass in the manipulation of objects might explain why the perceptual centre is close to the centre of mass. Indeed, the visual localization of the centre of mass allows for maximizing stability and minimizing force when grasping an object (Goodale et al., 1994; Iberall, Bingham, \& Arbib, 1986).

In this study, we investigate the ability of five-year old children to localize the centre of planar shapes. Because children manipulate objects at an early age, we expect them to estimate the centre of planar shapes in the vicinity of the centre of mass like adults. However, it is not known whether children use the same processes as the adults to localize the centre. To answer this question, we examined whether children and adults 
produced the same pattern of deviation from the centre of mass. In order to propose exactly the same task to 5-year old children and adults, we simplified Baud-Bovy and Soechting's experimental paradigm (2001) by asking the participants to mark with a pen the position of the centre of a triangle on a sheet of paper rather than on a touch screen.

MethodsParticipants

7 The participants were ten right-handed children aged from 4 years and 11 months to 5 years and 10 months (mean: 5 years and 6 months), and ten right-handed adults aged from 21 to 28 years (mean: 23 years and 9 months). The children were recruited from schools in France, and the adults were French undergraduate students. Their vision was normal and their handedness was assessed by their handed writing.

Experimental Procedure

Participants sat in front of a table and were similarly instructed in both groups to mark with a pen the centre of a triangle printed on a white sheet of paper ( 42 by $59.4 \mathrm{~cm}$ ) which was always presented in the portrait orientation. At the end of the experiment, the adults were asked what method, if any, they used to perform the task. The position of the mark relative to the centre of mass was coded manually by using a grid printed on a transparent sheet of paper with an accuracy of $\pm 1 \mathrm{~mm}$.

Stimuli
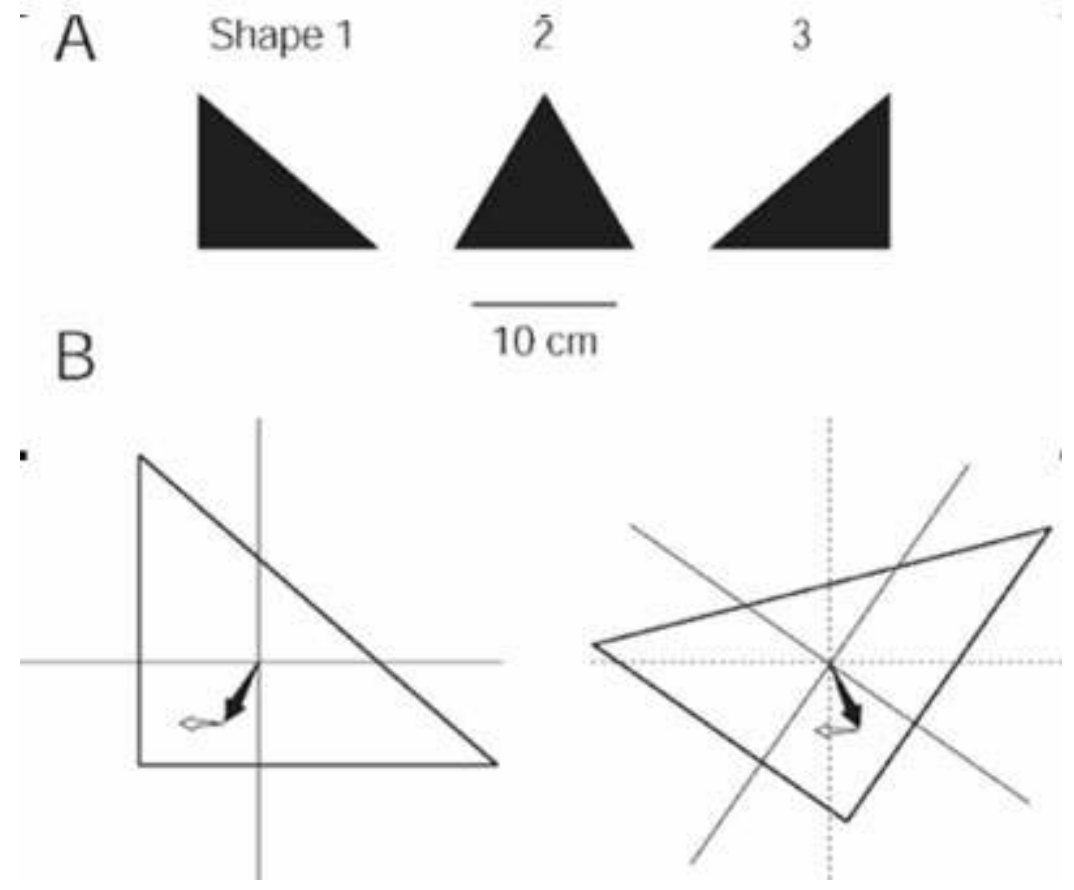

$9 \quad$ Figure 1. A: The two right triangles and the equilateral triangle (in the middle) used as stimuli. B: The object centred frame of reference (solid lines) rotates with the stimulus while the orientation of the fixed reference frame (dotted lines) remains invariant. According to the simple model described in the text, the response of the participant is the sum of two biases. The first bias, the offset (empty arrow), is fixed in the fixed reference frame while the second, the shape-effect (solid arrow), is fixed in the object reference frame.

10 The three tested triangles had the same height and area $\left(10.8 \mathrm{~cm}\right.$ and $\left.67.7 \mathrm{~cm}^{2}\right)$ and were presented once in each orientation (Figure 1A). The equilateral triangle was 
rotated from $0^{\circ}$ to $105^{\circ}$ in steps of $15^{\circ}$ around its centre of mass (8 orientations). The two right triangles were rotated from $0^{\circ}$ to $315^{\circ}$ in steps of $22.5^{\circ}$ around their centre of mass (16 orientations). The position of the triangle was randomly shifted on the paper sheet under the constraint that its centre of mass be located in an 8 by $8 \mathrm{~cm}$ square in the centre of the paper sheet. The presentation order was randomized for each participant.

Data Analysis

11 Figure 1B shows the two reference frames used in the analysis of the responses: The object coordinate system rotates with the triangle while the fixed coordinate system remained in the same position. Both reference frames are indistinguishable in the $0^{\circ}$ orientation. Their origin coincided with the true centre of mass. As in Baud-Bovy and Soechting (2001), the position of the perceptual centre is expressed as the sum of two biases (see Figure 1B): The shape-effect $(\xi, \eta)$ which denotes the perceived position of the centre relative to the centre of mass in the object reference frame and the offset $\left(X_{O}, Y_{O}\right)$ which represents a shift of the estimated position of the centre in the fixed frame of reference. Mathematically, the responses are modeled by the following expression:

$$
\left[\begin{array}{l}
x \\
y
\end{array}\right]=R(\alpha)\left[\begin{array}{l}
\zeta \\
\eta
\end{array}\right]+\left[\begin{array}{l}
x_{0} \\
y_{0}
\end{array}\right]+\left[\begin{array}{l}
\varepsilon_{x} \\
\varepsilon_{y}
\end{array}\right]
$$

where $(x, y)$ are the coordinates of the responses in the fixed reference frame, $\mathrm{R}(\mathrm{a})$ is a 2 by 2 rotation matrix, $(\xi, \eta)$ and $\left(X_{O}, Y_{O}\right)$ are the shape-effect and offset respectively, and $\left(\varepsilon_{\chi}, \varepsilon_{\gamma}\right)$ is a bivariate random variable with a null mean representing the variability of the response. The assumption that the shape effect $(\xi, \eta)$ is the same for all orientations can be verified by plotting the residuals $\left(\varepsilon_{\chi}, \varepsilon_{\gamma}\right)$ versus the orientation a. Any systematic deviation of the residuals from the origin indicates an orientation effect.

If the orientations $\mathrm{a}_{\mathrm{i}}$ are uniformly distributed and the experimental design is balanced, it is possible to compute the offset by averaging the positions of all responses in the fixed reference frame because the vectorial sum $\Sigma R\left(\alpha_{i}\right)[\xi, \eta]$ ' is zero over all trials. Under the same conditions, it is also possible to compute the shape-effect by averaging the positions of the responses in the object reference frame. The definition of the shape-effect is somewhat ambiguous for the equilateral triangle because the rotational symmetry of this shape makes the definition of an unique object reference frame impossible. Rather than selecting a reference frame arbitrarily, we defined the shape-effect to be null for this particular shape. 


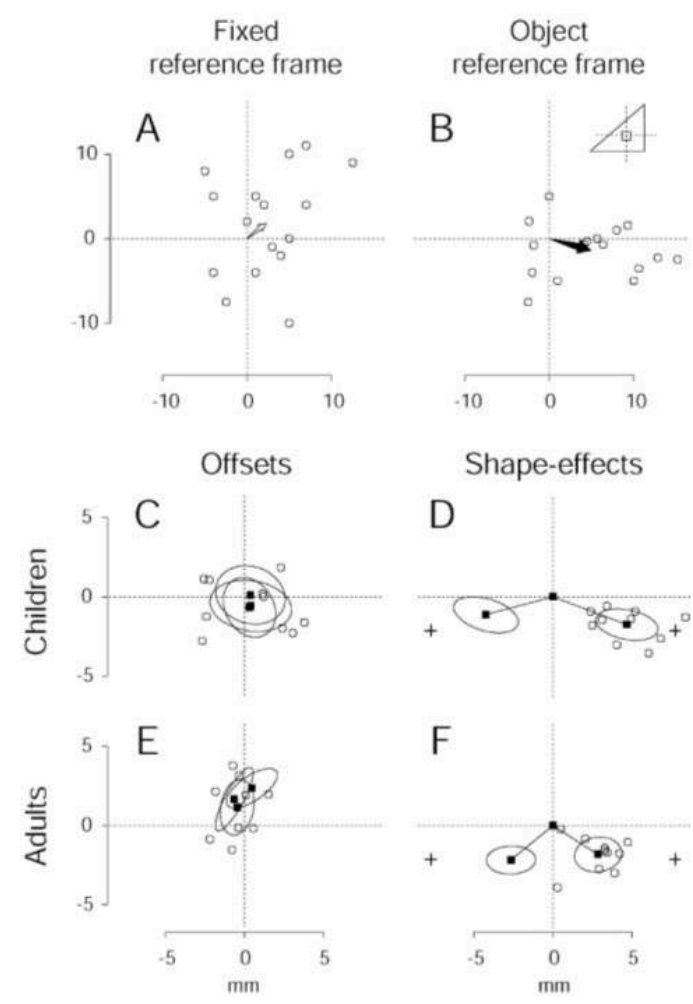

14 Figure 2. A, B: All the responses of a child for a particular shape are plotted in the fixed reference frame (Fig. 2A) and object reference frame (Fig. 2B). Each response was obtained with the right triangle in a different orientation (only the $0 \mathrm{E}$ orientation is plotted in the inset of Figure 2B). The origin of both reference frames coincides with the center of mass. The plot area corresponds to the square in the center of the triangle in the inset. The empty arrow points toward the centroid of the responses in the fixed reference frame (the offset) while the solid arrow points toward the centroid of the responses in the object reference frame (the shape-effect, see Methods). C, D: Distribution of the offsets (Fig. 2C) and shape effects (Fig. 2D) for the children. The centers (solid dots) of the ellipses of confidence correspond to the mean offset or shape-effect for each triangle (data from all children are pooled, $\mathrm{N}=10$ ). The shapeeffect of the equilateral triangle is zero by definition. The empty dots indicate the mean response for each child obtained with the same right triangle as in Figs. 2A and 2B. The two crosses in Fig. 2D indicate the position of the center of the inscribed circles for the two right triangles. E, F: Distribution of the offsets and shape-effects for the adults.

Results

The children, like the adults, understood and performed the task without problems. To illustrate how the offset and shape-effect are computed, we show all responses of one child in the fixed frame of reference (Figure 2A). The offset (i.e., the average position of the responses in the fixed reference frame, see Methods) represents a bias in the localization of the centre of the figure that is independent of the orientation of the triangle. In Figure 2B, the same data points are represented in the object reference frame. The centroid of the responses in this reference frame corresponds to the shapeeffect. This figure shows that the data points are more grouped when they are represented in the object reference frame. This observation suggests that this child tended to deviate systematically from the true centre of mass toward the right angle of this triangle. The scatter around the centroid represents the intrinsic variability of the 
response when the participant estimates the position of the centre of the shape and a possible orientation-effect (see later).

The empty dots in Figures $2 \mathrm{C}$ and $2 \mathrm{D}$ represent the offsets and shape-effects of all children obtained with the same triangle as above. The scatter of the dots in Figure $2 \mathrm{C}$ shows that the offsets varied in direction and magnitude across children. In contrast, the position of the dots in Figure 2D indicates that the all children tended to localize the centre in the lower right part of the triangle, in direction of the right angle. To compare the distribution of the offsets or of the shape-effects across shapes, we computed the $95 \%$ ellipse of confidence around the mean value for each shape $(\mathrm{N}=10)$. The centre of each ellipse represents the mean position of the offsets (Figure 2C) or of the shape-effect (Figure 2D) for a particular triangle while its size represents an estimate of the between-subject variability. The overlap of the three ellipses in Figure $2 \mathrm{C}$ indicates that the offsets were similar for all triangles. This observation held true not only in average but also for each child taken separately (data not shown). In other words, the positions of the offsets are similar across shapes for each child even though there are marked differences in their position across children. The two ellipses in Figure 2D represent the distribution of the shape-effects for the two right triangles. The positions of the two ellipses are mirror symmetric with respect to the vertical axis, a finding to be expected given the symmetry of the two right triangles. For adults and children alike, the ellipses are centreed approximately at mid-distance between the centre of mass and the centre of the inscribed circle as in Baud-Bovy and Soechting (2001). The shape-effect of the equilateral triangle is null by definition.

Table 1. Magnitude of the Offsets, Shape-Effects and Residuals

\begin{tabular}{l|c|c|c|c}
\hline & Shape & Offset & Shape-effect & Residuals \\
\hline \multirow{3}{*}{ Adults } & 1 & 1.8 & 3.4 & 3.7 \\
& 2 & 2.4 & 0 & 4.2 \\
\hline \multirow{3}{*}{ Children } & 3 & 1.2 & 3.4 & 3.5 \\
\cline { 2 - 5 } & 1 & 0.3 & 4.6 & 4.9 \\
& 2 & 0.7 & 0 & 5.4 \\
\hline
\end{tabular}

The results obtained with adults were very similar to those obtained with children (Figures $2 \mathrm{E}$ and $2 \mathrm{~F}$ ). In particular, the shape-effects of the two right triangles were all located a few millimeters off the centre of mass in the direction of the right angle. Table 1 reports the distance from the origin of the average offset and shape effect position for each shape and group (see solid dots in Figure 2). The magnitude of the offset was smaller that the magnitude of the shape-effect except for the equilateral triangle in both groups. There were however several small quantitative differences between the two groups. While the offsets of children were scattered around the centre of mass, the offsets for the adults were located, on average, slightly above the centre of mass (Figure 2E). In addition, the magnitude of the shape-effect (distance from the centre of mass) was, on average, slightly larger for children than for adults (4.8 vs 3.4 $\mathrm{mm}$ ) and represented about $3-4 \%$ of the basis of the triangles in the standard orientation $(125 \mathrm{~mm})$.

18 These observations were confirmed by a two-way MANOVA with group as betweensubject factor and shape as within-subject factor. The coordinates of the responses in the fixed frame of reference were different between the two groups 
(Wilks $\left.=0.964, \lambda_{(2,793)}=14.959, \mathrm{P}<0.001\right)$. Neither the shape nor the interaction was statistically significant in this analysis. A two-way MANOVA conducted with the coordinates of the responses in the object reference frame indicated that both the shape factor $\left(\mathrm{W}=0.667, \lambda_{(2,1586)}=88.83, \mathrm{P}<0.001\right)$ and the shape $x$ group interaction $\left(\mathrm{W}=0.951, \lambda_{(4,1586)}=10.131, \mathrm{P}<.001\right)$ were statistically significant. The group factor was not statistically significant.

The average size of the residuals is comparable to the size of the effects reported (see Table 1). However, Part of this variability might be due to an effect of the stimulus orientation on the perceptual centre. Such an effect might also explain why the residuals were, on average, larger for the equilateral triangle than for the two right triangles (see below). This observation is indeed surprising given the fact that the variability of the response is usually smaller for symmetrical than asymmetrical figures (Baud-Bovy \& Soechting, 2001; Bingham \& Muchisky, 1993b; Davi et al., 1992). Note that we cannot estimate the variability of the response for each participant independently of the orientation of the stimulus because the experimental design does not include replications.

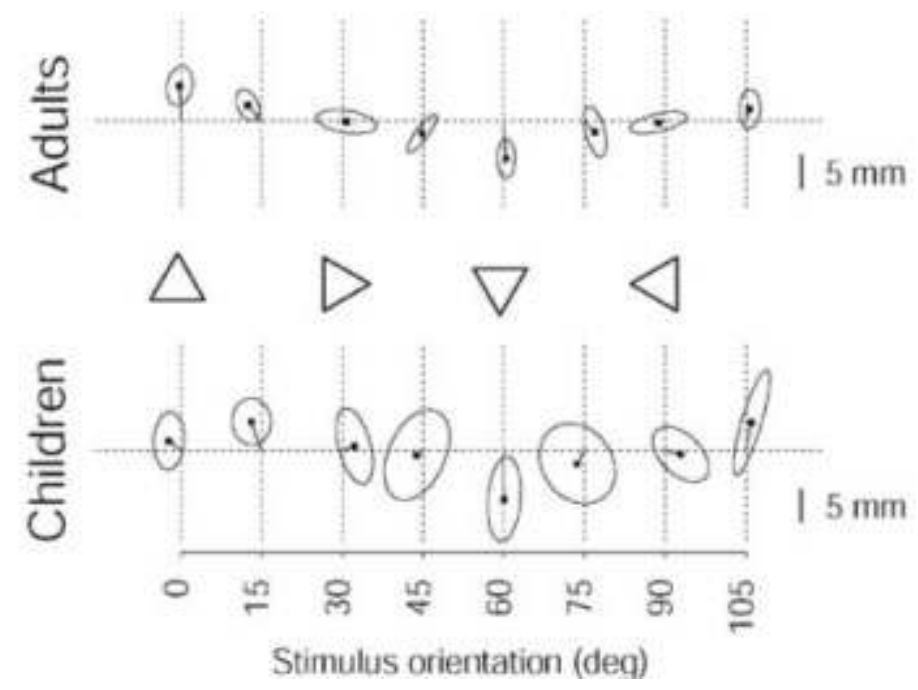

Figure 3. Plot of the residuals of the model against the orientation of the stimulus. The 95\% ellipses of confidence are computed by pooling together the residuals from all participants in each group $(\mathrm{N}=10)$. The fact that the ellipses are not centred on the origin shows an effect of the orientation on the perceptual centre of the equilateral triangle.

The model used so far in the analysis of the responses assumes that the offset and shape-effect are the only biases that determine the location of the perceptual centre relative to the centre of mass. According to this model, the residuals should be centreed on the origin for all orientations. To check this assumption, we plotted the residuals against the orientation for each shape separately. We computed a $95 \%$ ellipse of confidence around the mean value for each orientation after pooling data from all participants in each group $(\mathrm{N}=10)$. Figure 3 shows a clear orientation effect for the equilateral triangle (nothing comparable was observed for the right triangles). Participants in both groups tended to perceive the centre of the equilateral triangle in different position depending on its orientation. The perceptual centre was located above the centre of mass in the $0^{\circ}$ orientation and below it in the $60^{\circ}$ orientation. In 
other words, the perceptual centre was shifted toward the vertex opposite the horizontal edge of the equilateral triangle. No similar shift occurred when one of the edges of the triangle was vertical (see $30^{\circ}$ and $90^{\circ}$ orientations).

3. Discussion

The most striking result of this study is the degree of similarity of the responses between the young children and the adults. As hypothesized, we found that children, like adults, performed the task accurately and seemingly effortlessly. On average, the deviations from the centre of mass represented less than $5 \%$ of the object size for both groups. In addition, the pattern of deviations from the centre of mass was qualitatively similar in both groups: 1) The analysis of the responses in the object reference frame revealed that the shape-effects were located at mid-distance between the centre of mass and the centre of the inscribed circle for almost all participants in both groups (Baud-Bovy \& Soechting, 2001). 2) Although the offsets of the adults were located slightly above the children's ones, they did not depend on the shape and did not stray far from the centre in either group. 3) Finally, the analysis of the residuals showed that, for the equilateral triangle, the perceptual centre depended upon the orientation of the triangle. For children and adults alike, the perceptual centre was shifted toward the vertex that opposed the horizontal edge of the triangle (see Davi, 1994; Davi \& Proffitt, 1993; for a discussion of a possibly related effect).

In Baud-Bovy and Soechting (2001), we concluded that the shape-effect corresponded to the perceived position of the centre of mass of planar shapes while the offset reflected some additional source of error related to the mode of response. The offset positions in the present study differed from the leftward oriented offsets observed when the participants responded by touching a vertically oriented touch screen with their fingertip but resembled those obtained when participants used a computer mouse to respond in our previous study. Bingham and Muchisky (1993a) have argued that this leftward bias of the offset increases the stability of the grasp. According to this view, the lack of leftward bias observed when the object is presented in the horizontal plane or when the computer mouse is used can be explained by the fact that stability plays a lesser role in these conditions.

Because it is not clear how the shape-effect might improve the stability of the grasp, we believe that this effect is best explained in terms of a trade-off between performance and computational complexity (see Cruse \& Brüwer, 1987, Haggard \& Richardson, 1996, and Soechting \& Flanders, 1989, for other examples of such trade-offs in motor control). In other words, the small but systematic deviations toward the centre of the inscribed circles in either group is explained by the fact that such a bias would reduce the computational complexity of estimating the position of the centre of mass. In BaudBovy and Soechting (2001), we proposed that the visual localization of the centre of a 2D shape might involve a process in which an abstract representation of a filled polygon is filtered with a circular filter such as a difference of Gaussians (Campbell \& Robson, 1968; Jones \& Palmer, 1987).

The proximity of the perceptual centre to the centre of mass, a crucial physical variable for the manipulation of objects, suggests that perceptual processes might tap motor competence to perform this task (Bingham \& Muchisky, 1993ab; Baud-Bovy \& Soechting, 2001). However, it is possible that the visual localization of the centre of a 2D shape involves different processes and/or visual cues than those used when grasping an object since it is a perceptual rather than a motor task (Goodale et al., 1994; Smeets, 
Brenner, de Grave, \& Cuijpers, 2002). It remains to be seen whether one could accurately infer the estimated position of the centre of mass in a motor task, and whether such an estimate would present the same biases as in a perceptual task.

In any case, these results support the idea that children process perceptual shape information in the same way as adults. For example, it is known that vertical symmetry plays a similar organizational role in four-month old infants (Fisher, Ferdinandsen, \& Bornstein, 1981), in older children (e.g., Bornstein \& Stiles-Davis, 1984) and in adults (e.g., Royer, 1981). In other words, the centre, like vertical symmetry, could play the same structuring role in the visual analysis of 2D shapes in children and adults.

Several lines of evidence suggest also that the visual localization of the centre of planar shapes is based on low-level highly automatized sensorimotor processes. First, the similarity of the responses in both groups indicates that the processes involved in this task are in place from the age of five if not earlier. The age of the children excludes the possibility that this similarity might stem from notions acquired at school. Instead, these results of this study suggest that such ability must either be based on some inherent structure, or be acquired through early sensori-motor experiences such as the grasping and manipulation of objects. Second, the adults also did not ask any questions about the instructions during the experiments in spite of the fact that the notion of the centre of a triangle is ambiguous. In fact, their performance did not seem to be related to the strategy that they reportedly use or to their response time (Baud-Bovy \& Soechting, 2001). Third, the fact that we obtained the same results in spite of a change in the instruction set (i.e., after asking participants to identify the position of the "centre of the shape" instead of the position of the "point of equilibrium" as in our previous study) suggests that the underlying processes are cognitively impenetrable (Pylyshyn, 1999).

Acknowledgments

This work was supported by grants of the National Centre of Scientific Research (CNRS). We thank Anne Mallet for collecting the data with the children and Prof. Paolo Viviani for his comments on this manuscript.

\section{BIBLIOGRAPHY}

Baud-Bovy, G., \& Soechting, J. (2001). Visual localization of the centre of mass of compact, asymmetric, two-dimensional shapes. Journal of Experimental Psychology: Human Perception and Performance, 27, 692-706.

Beghi, L., Vicario, G., \& Zanforlin, M. (1983). The perceptual centre of visual configurations. Atti e Memorie della Accademia Paravina di Scienze. Lettere ed Arti: Parte III, Classe di Scienze Morali, 95, 133-148.

Bingham, G. P., \& Muchisky, M. M. (1993a). Centre of mass perception and inertial frames of reference. Perception \& Psychophysics, 54, 617-632. 
Bingham, G. P., \& Muchisky, M. M. (1993b). Centre of mass perception: Perturbation of symmetry. Perception \& Psychophysics, 54, 632-639.

Bornstein, M., \& Stiles-Davis, J. (1984). Discrimination and memory for symmetry in young children. Developmental Psychology, 20, 637-649.

Campbell, F. W., \& Robson, J. G. (1968). Application of Fourier analysis to the visibility of gratings. Journal of Physiology (London), 197, 551-566.

Cruse, H., \& Brüwer, M. (1987). The human arm as a redundant manipulator: The control of path and joint angles. Biological Cybernetics, 57, 137-144.

Davi, M. (1989). Il centro percettivo di figure piane. Giornale Italiano di Psicologia, 16, 45-60.

Davi, M., Thomas Doyle, M. A., \& Proffitt, D. R. (1992) The role of symmetry in determining perceived centres within shapes. Perception \& Psychophysics, 52, 151-160.

Davi, M., \& Proffitt, D. R. (1993). Frames of reference and distinctive figural characteristics affect shape perception. Journal of Experimental Psychology: Human Perception and Performance, 19, 867-877.

Davi, M. (1994). A model for identifying the perceptual centre of polygones. Perception, 23, 1085-1095.

Fisher, C., Ferdinansen, K, \& Bornstein, M. (1981). The role of symmetry in infant form discrimination. Child Development, 52, 457-462.

Goodale, A. M., Meenan, J. P., Bulthoff, H. H., Nicolle, D. A., Murphy, K. J., \& Racicot, C. I. (1994) Separate neural pathways for the visual analysis of object shape in perception and prehension. Current Biology, 4, 604-6120.

Haggard, P., \& Richardson, J. (1996). Spatial patterns in the control human arm movement. Journal of Experimental Psychology: Human Perception and Performance, 22, 42-62.

Iberall, T., Bingham. G. P., \& Arbib, M. A. (1986). Opposition space as a structuring concept for the analysis of skilled hand movements. In H. Heuer \& C. Fromm (Eds.), Generation and modulation of action patterns (pp. 158-173). Berlin: Spinger-Verlag.

Jones, J. P., \& Palmer, L. A. (1987). An evaluation of the two-dimensional Gabor filter model of simple receptive fields in cat striate cortex. Journal of Neurophysiology, 58, 1233-1258.

Proffitt, D. R., Thomas, M. A., \& O’Brien, R. G. (1983). The roles of contour and luminance distribution in determining perceived centres within shapes. Perception \& Psychophysics, 33, 63-71.

Pylyshyn, Z. (1999). Is vision continuous with cognition? The case for cognitive impenetrability of visual perception. Behavioral and Brain Science, 22, 341-365.

Royer, F. (1981). Detection of symmetry. Journal of Experimental Psychology: Human Perception and Performance, 7, 1186-1210.

Smeets, J. B. J., Brenner, E, de Grave, D. D. J., Cuijpers, R. H. (2002) Illusions in action: consequences of inconsistent processing of spatial attributes. Experimental Brain Research, 147:135-144.

Soechting, J.F., \& Flanders, M. (1989). Errors in pointing are due to approximations in sensorimotor transformations. Journal of Neurophysiology, 62, 582-594.

Vos, P. G., Bocheva, N., Yakimoff N., \& Helsper, E. (1993). Perceived location of two-dimensional patterns. Vision Research, 15, 2157-2169. 


\section{ABSTRACTS}

The ambiguity of the notion of centre for most shapes raises the question of whether children perceive the centre of a shape in the same place as adults. To answer this question, we asked fiveyear old children and adults to mark the centre of a triangle with a pen. The responses of children and adults were strikingly similar. First, the two groups perceived the centre of right triangles at mid-distance between the centre of mass and the centre of the inscribed circles. Second, the perceptual centre of the equilateral triangle was shifted toward the vertex that opposed the horizontal edge of the triangle for children and adults alike. These results suggest that the visual localization of the centre of triangles would depend on low-level sensori-motor processes that are in place since at least the age of five.

La notion de centre est ambiguë pour la plupart des formes géométriques. Pour étudier cette question, nous avons demandé à des enfants âgés de cinq ans et à des adultes de marquer le centre d'un triangle sur une feuille de papier. Les réponses des deux groupes étaient très similaires. Premièrement, les deux groupes tendaient à percevoir le centre du triangle rectangle à mi-distance entre le centre de masse et le centre du cercle inscrit. Deuxièmement, la position du centre du triangle équilatéral était déplacée en direction de la pointe opposée au côté horizontal. Ces résultats suggèrent que la localisation visuelle du centre de triangles dépend de processus perceptifs de bas-niveau, déjà en place à l'âge de cinq ans.

\section{INDEX}

Keywords: Visual perception, perceptual centre, centre of mass, planar geometry, grasping

\section{AUTHORS}

\section{GABRIEL BAUD-BOVY}

Faculty of Psychology, UHSR University, via Olgettina 58, Milan 20132, Italy baud-bovy.gabriel@hsr.it

\section{EDOUARD GENTAZ}

National Centre of Scientific Research (CNRS), Laboratory of Cognition and Development University René Descartes Paris V, France. 